\title{
Advances in heart failure pharmacotherapy and cardio-vascular prevention: reports from The Congress of European Society of Cardiology 2020
}

\author{
Piotr Hamala (D), Jarosław D. Kasprzak (D) \\ Department of Cardiology, Medical University of Lodz, Poland
}

\section{Introduction}

Due to the coronavirus disease 2019 (COVID-19) pandemic, this year's European Society of Cardiology (ESC) Congress was held online. Despite the changed form of the Congress, its programme did not lack interesting reports on international clinical trials completed over the past year. This year was particularly abundant in important, positive results that will probably change our practical approach to treatment. Below is a subjective selection of the most interesting results presented.

\section{LoDoCo2: low-dose colchicine-2}

Atherosclerotic process progression, destabilization and atherosclerotic plaque rupture are associated with activation of the inflammatory process involving interleukins interleukin (IL) $1 \beta$ and IL-18, among others. With its anti-inflammatory effect, colchicine blocks pathophysiological pathways leading to the production of these interleukins, hence the hypothesis of a beneficial effect of colchicine in chronic coronary artery disease [1]. The conducted study followed the pilot LoDoCo, which revealed that treatment with colchicine provided beneficial effects in secondary prevention of cardiovascular events, but due to the small size of the study group $(n=532)$ and the lack of a double-blind sample, the results had to be confirmed in a study that provided more powerful evidence [2]. LoDoCo2 was a randomized, double-blind placebo-controlled trial that involved a total of 5,522 patients aged 35 to 82 . The following inclusion criteria were applied: chronic coronary artery disease confirmed based on coronary angiography or computed tomography (Agatston index $>400 \mathrm{HU}$ ), lack of exacerbations of coronary artery disease for $\geq 6$ months. The exclusion criteria included: moderate and severe renal impairment, severe heart failure, a severe valvular heart defect and a history of an adverse reaction during colchicine treatment. The patients included in the study were divided into two groups. The first group received colchicine orally, at a dose of $0.5 \mathrm{mg} /$ day $(n=2,762)$ and the second group received placebo ( $n=2,760$ patients). The median follow-up time was 29 (12-64) months, and the primary composite endpoints were: myocardial revascularisation due to ischaemia, ischaemic stroke, myocardial infarction, and death from cardiovascular causes. There were 8 secondary endpoints, some of them composite. The first one, crucial, included: cardiovascular death, myocardial infarction, and ischaemic stroke. The second included myocardial infarction and myocardial revascularization due to ischaemia. The third included cardiovascular death, myocardial infarction. The other, hierarchically ranked, secondary endpoints were myocardial infarction, ischaemic stroke, death (from any cause), and death from cardiovascular causes.

The primary composite endpoint concerned 264 patients in the placebo group and 187 in the colchicine group. The risk of the occurrence of endpoint in the group treated with colchicine was significantly reduced compared to the placebo group - by 31 per cent [95 per cent confidence interval (Cl): $0.57-0.83, \mathrm{p}<0.001$ ]. Similarly, a $28 \%$ decrease was observed in the case of the secondary endpoint risk (95\% $\mathrm{Cl} 0.57-0.92, \mathrm{p}=0.007)$. There were no differences between groups in terms of the frequency of adverse effects in response to both treatments. In conclusion, in patients with the chronic coronary syndrome, colchicine

Adress for correspondence: Piotr Hamala MD, Katedra i Klinika Kardiologii, Uniwersytet Medyczny w Łodzi, ul. Kniaziewicza 1/5, 91-347 Lodz, Poland, phone/fax +48 4265399 09, e-mail: piotrhamala@gmail.com

This article is available in open access under Creative Common Attribution-Non-Commercial-No Derivatives 4.0 International (CC BY-NC-ND 4.0) license, allowing to download articles and share them with others as long as they credit the authors and the publisher, but without permission to change them in any way or use them commercially. 
added to standard treatment was safe and improved the efficacy of secondary prevention of cardiovascular events, becoming a strong candidate to be added to the palette of preventive drugs used in chronic coronary syndromes [3].

\section{EMPEROR-Reduced}

EMPEROR-Reduced, which investigated the usefulness of empagliflozin added to standard treatment in a group of patients with heart failure and reduced ejection fraction, was a highly anticipated trial. The study group consisted of 3,730 patients from 20 countries with heart failure and left ventricular ejection fraction $(E F) \leq 30$ per cent, or with $\mathrm{EF} \geq 30$ per cent with high values of N-terminal pro-B-type natriuretic peptide (NT-proBNP). The group included patients with and without type 2 diabetes. The primary endpoint included: hospitalization due to heart failure and death from cardiovascular causes. The first secondary endpoint was the total number of hospitalizations for cardiovascular reasons during the observation and the second secondary endpoint was the rate of glomerular filtration reduction. The median observation time was 16 months. Regardless of their diabetes status, 1,863 patients were randomized into the empagliflozin group [10 $\mathrm{mg} /$ day per os (p.o.)], and 1,867 patients were receiving a placebo. In a survival curve analysis for the primary endpoint, the endpoint was reached by 462 patients in the placebo group and 361 in the study group, which meant that the risk of cardiovascular death or hospitalization due to heart failure was reduced by 25 per cent ( $95 \% \mathrm{Cl}$ : 0.65-0.86, $p<0.0001)$. When the primary endpoints were compared separately, the risk reduction was as follows: for hospitalization for heart failure, the $\mathrm{HR}$ and $95 \% \mathrm{Cl}$ were 0.69 and (0.69-0.81); $p<0.0001$, and for death from cardiovascular causes -0.92 and $(0.75-1.12) ; p=$ not statistically significant (NS). From the perspective of reducing the risk of endpoints, a favourable effect in the study group was also observed for secondary endpoints - in particular, a significant difference in the rate of decrease in estimated glomerular filtration rate (eGFR) was observed between groups. The decrease in eGFR was $4.2 \mathrm{~mL} / \mathrm{min} / 1.73 \mathrm{~m}^{2}$ in the placebo group and $0.9 \mathrm{~mL} / \mathrm{min} / 1.73 \mathrm{~m}^{2}$ in the study group $(p<0.0001)$. The above observation corresponds with the results of the analysis of the risk of composite renal endpoint, the components of which included: eligibility for chronic dialysis, renal transplantation, as well as a profound and persistent decrease in eGFR. During the analysis of the occurrence of the above composite endpoint, it was observed that in the study group the risk decreased (HR 0.50; 95\% Cl: 0.32-0.77, p < 0.001). The authors compared the above results with the results of the DAPA-HF study (a randomized double-blind study, a comparison of treatment with dapagliflozin with placebo in patients with heart failure, $n=2,373$, the results of which were presented at the European Cardiological Association Congress of 2019 in Paris [4]. Patients from EMPEROR-Reduced had lower EF $27.7 \pm 6.0$ versus 31.2 $\pm 6.7 \%$, lower eGFR $61.8 \pm 21.7$ versus $66.0 \pm 19.6 \mathrm{~mL} / \mathrm{min}$ and higher NT-proBNP 1926 (1,153-3,525) versus 1,887 $(1,077-3,429) \mathrm{pg} / \mathrm{mL}$. The risk reduction for the primary endpoint in the study groups was the same in both studies (25\%), but the EMPEROR-Reduced patients had a lower risk reduction of cardiovascular death than the DAPA-HF patients but a stronger risk reduction renal composite endpoint [HR and $95 \% \mathrm{Cl}$, respectively: $0.50(0.32-0.77)$ vs. $0.71(0.44-1.16)]$. These differences in survival analyses may be explained by the differences in the severity of heart failure between the study groups of both studies. Due to such aspects as the lack of homogeneity in the definition of the remaining endpoints, it is difficult to precisely compare both trials. In order to gain a more thorough understanding of the differences between empagliflozin and dapagliflozin, it would be necessary to conduct a head-to-head study of both molecules [5] - however, at present, both studies provide strong support for the inclusion of sodium-glucose co-transporter 2 (SGLT2) inhibitors as useful drugs in heart failure, regardless of diabetic status.

\section{DAPA-CKD}

Nephroprotection is an important goal of prevention therapies that is difficult to achieve. The DAPA-CKD study aimed to compare dapagliflozin treatment (10 mg p.o./day) with placebo in terms of its effect on the risk of cardiovascular and renal events in patients with chronic kidney disease. The study involved 4,304 patients in 21 countries. The median observation time was 2.4 years. The inclusion criteria were as follows: age $>18$ years, eGFR within the range $25-75 \mathrm{~mL} / \mathrm{min} / 1,73 \mathrm{~m}^{2}$, albumin/creatinine ration within the range from 200 to $5,000 \mathrm{mg} / \mathrm{g}$, tolerance for the maximum dose of angiotensin converting-enzyme inhibitor angiotensin receptor blocker (ACEl/ARB) for $\geq 4$ weeks (if it is not contraindicated). The above group included both patients with and without type 2 diabetes.

Exclusion criteria were: diagnosis of type 1 diabetes mellitus, polycystic kidney disease, lupus nephropathy, anti-neutrophil cytoplasmic vasculitis (ANCA) and undergoing immunosuppressive therapy within 6 months before study enrollment. The study group was compared with a control group that matched it in terms of age, sex, ethnicity, distribution of type 2 diabetes, systolic blood pressure, glomerular filtration, the albumin/creatinine ratio and proportion of participants taking ACEI/ARB.

The primary composite endpoint included: permanent reduction in glomerular filtration by $\geq 50 \%$, end-stage kidney disease, death related to kidney disease and death related to cardiovascular causes. It was reached by 312 patients in the placebo group and 197 patients receiving 
dapagliflozin. In the study group, the risk of the primary composite endpoint was reduced by 39 [hazard ratio (HR) 0.61; 95\% Cl: 0.51-0.72, $\mathrm{p}<0001$ ].

Another element of the study was the analysis of the composite primary renal endpoint - it was defined in the same way as the previous one, but excluding death related to cardiovascular causes. The above endpoint was reached by 243 patients in the placebo group and 142 individuals in the group receiving dapagliflozin. In the study group, the relative risk in the incidence of the primary composite renal endpoint was reduced by $44 \%$ (HR 0.56; $95 \% \mathrm{Cl}$ : 0.45-0.68, $p<0.0001$ ).

Beneficial effects of dapagliflozin were observed in subgroups of patients with and without type 2 diabetes [HR and $95 \% \mathrm{Cl}$, were respectively 0,64 (0.52-0.79) vs. $0.50(0.35-0.72) p=0.24]$, which means that the diagnosis of type 2 diabetes does not affect the beneficial effects of dapagliflozin treatment.

Hospitalization due to heart failure and death due to cardiovascular causes constituted the secondary end point. It was achieved by 138 patients in the placebo group and 100 patients in the dapagliflozin group (HR 0.71; 95\% Cl: $0.55-0.92, p=0.0089$ ). During the analysis of the risk of total mortality, the researchers observed that the relative risk was reduced by $31 \%$ (HR 0.69; 95\% $\mathrm{Cl}$ : 0.53-0.88, $p=0.0035)$.

Dapagliflozin tolerance was not worse than that of the placebo. The proportions of discontinuation of treatment due to intolerance were $12.8 \%$ in the study group and $14.4 \%$ in the control group ( $p=0.16$ ). In the study subgroup that included patients without type 2 diabetes, there were no episodes of hypoglycaemia. In conclusion, the study provides evidence of further benefits provided by dapagliflozin treatment, not only among patients with diabetes and heart failure but also among patients with renal disease [6].

\section{BRACE-CORONA}

The COVID-19 pandemic motivated the world of science to rapidly collect scientific data. One of the controversies associated with the coronavirus was the fact that the membrane molecule of angiotensin-converting enzyme 2 (ACE2) is a receptor for severe acute respiratory syndrome coronavirus 2 (SARS-CoV-2). Since inhibition of the renin-angiotensin system is associated with increased expression of ACE2 on the plasma membrane, at the beginning of the pandemic there were concerns about the adverse effect of ACEI/ARB on the risk and course of COVID-19. Although these concerns were not confirmed by experts [6, 7], in March Brazilian researchers designed and very quickly carried out a prospective study on the topic in question. The BRACE CORONA study aimed to clarify whether discontinuation or continuation of ACEI treatment affected the prognosis of patients hospitalised to due COVID-19. It was a randomized study that included 659 patients. The observation time was 30 days and the mean age of patients was 55; 41 per cent of the participants were women. Inclusion criteria included: age $\geq 18$ years, hospitalisation due to COVID-19, history of chronic use of ACEI/ARB. Exclusion criteria included: history of heart failure exacerbation within the past year, treatment with $\geq 4$ antihypertensive medications, treatment with sacubitril/valsartan. The primary parameter analysed to assess prognosis in this group was the number of days alive and out of the hospital, calculated throughout the observation. There were no significant statistical differences in the number of days alive and days out of hospital - on average, they amounted to: $21.9 \pm 8.0$ days in the group where ACEI/ARB was discontinued and 22.9 \pm 7.1 days in the group where the treatment was continued (mean ratio 0.95; 95\% $\mathrm{Cl}: 0.90-1.01, \mathrm{p}=0.09$ ). All-cause mortality constituted the secondary endpoint. There was no difference in the proportion of the occurrence of the above endpoint in the observed subgroups. In the group of patients who continued the ACEI/ARB treatment, all-cause mortality reached $2.8 \%$ and in the group of patients who discontinued the ACEI/ARB treatment $-2.7 \%$, relative risk (HR 0.97; 95\% Cl: 0.38-2.52, p = 0.95).

The discussion emphasized the relatively young age of the study group (mean age 55 years) as a factor that may result in differences in the prognosis of patients who are on ACEI/ARB therapy, the lack of comparison separately of the effects of therapy with each group of ACE and ARB drugs (different mechanisms of action of each drug group), and the relatively short follow-up time. In conclusion, the above study did not find evidence confirming an adverse prognostic effect of the ACEI/ARB treatment in patients hospitalized due to COVID-19 [8].

\section{RATE-AF}

The prospective, randomized RATE-AF study was conducted to compare long-term beta-blocker and digoxin treatment in patients with sustained atrial fibrillation (AF) and symptoms of heart failure. Inclusion criteria were: age $\geq 60$ years, sustained AF, symptoms of New York Heart Association (NYHA) class II (or higher) heart failure, and the need for the implementation of ventricular rate control treatment. Exclusion criteria were: heart rate $<60 / \mathrm{min}$, history of poor tolerance for heart rate-lowering therapy, and history of diagnosed heart failure. Observation of the effects of the introduced treatment was performed with the use of the Quality-of-Life Assessment Questionnaire SF-36 (SF-36) in the $6^{\text {th }}$ month of the observation. Heart rate, NYHA score and NT-proBNP levels were also assessed in the $6^{\text {th }}$ and $12^{\text {th }}$ of the observation, and quality of life left ventricular ejection fraction and incidence of adverse effects were assessed again at 12 months. 
A group of 160 patients, using a minimal randomization algorithm (by sex and EHRA class), were divided into two groups, treated to achieve heart rate control with digoxin $(n=80)$ or a beta-blocker (bisoprolol) $(n=80)$. The mean age was $76 \pm 8$ years; $46 \%$ of the group was female.

When the results for the primary parameter, quality of life at the $6^{\text {th }}$ month of follow-up, were analysed, no differences were observed between the groups ( $p=0.3$ ). At the $12^{\text {th }}$ month follow-up, some questionnaire items indicated better quality of life among patients taking digoxin e.g., SF36-GH (general sense of health, $p=0.049$ ), SF36-PF (physical functioning, $p=0.05$ ). There was no difference in the reduction of ventricular rate. NYHA class at 12-month follow-up decreased in the digoxin group from a mean of 2.4 to 1.5, and in the beta-blocker group from 2.4 to 2.0 ( $p<0.001$ ). NT-proBNP levels at 12 -month follow-up decreased in the digoxin group from a mean of 1,095 to $960 \mathrm{pg} / \mathrm{mL}$ and increased in the beta-blocker group from 1041 to $1250 \mathrm{pg} / \mathrm{mL}(p=0.005)$. Left ventricular ejection fraction in the 12th month of follow-up increased in the digoxin group from an average of $56.2 \%$ to $59.7 \%$, and in the beta-blocker group from $57.6 \%$ to $59.8 \%(p=0.45)$. Ejection fraction adverse effects were more frequently observed in the beta-blocker treatment group 142 cases versus 29 in the digoxin group. These data suggest a more favourable effect and greater safety of digoxin for chronic ventricular rate control therapy in patients with fixed atrial fibrillation compared with bisoprolol therapy, in contrast to current recommendations.

\section{EAST-AFNET 4}

Despite optimal therapy in patients with AF, complications of this disease such as exacerbation of heart failure, acute coronary syndrome, and ischaemic stroke are still observed. To date, studies conducted have not proven the superiority of heart rate control over ventricular rate control $[8,9]$. Given the above, the following study was designed to address the question of whether early cardiac rhythm control improves prognosis compared with typical therapy. This was a prospective, randomized study conducted in 11 Central and Western European countries (including Poland). Inclusion criteria were: $\mathrm{CHA}_{2} \mathrm{DS}_{2} \mathrm{VASc} \geq 2$, the occurrence of ECG-documented AF attack $\leq 1$ year. Patients were randomized to two treatment strategies. The first strategy (early rhythm control, $n=1,395$ ) consisted of early initiation of antiarrhythmic drugs, anticoagulation, or early eligibility for AF ablation, and, in the event of a recurrent AF attack, eligibility for repeat ablation or modification of antiarrhythmic pharmacotherapy. In the second group ( $n=1,394)$, heart rate control was implemented only for symptomatic patients previously receiving optimal heart rate control therapy.
The primary endpoint was composite and included worsening heart failure, acute coronary syndrome, stroke, and cardiovascular death. The second item spent assessed was the number of days in the hospital per year.

The median follow-up was 5.1 (3.8-6.4) years. The primary endpoint was reached by 249 patients in the early rhythm control strategy group and 316 patients in the second group. A serious adverse event occurred in 68 (4.9\%) patients in the early rhythm control strategy group compared to $19(1.4 \%)$ patients in the other group ( $p<0.001)$. Stroke occurred in 40 (2.9\%) patients in the early rhythm control strategy group, compared to 62 patients $(4.4 \%)$ in the second group ( $p=0.03$ ) [10].

When comparing the groups in terms of the number of nights spent in the hospital, no differences were observed. Patients in the early cardiac rhythm control strategy group spent an average of $5.8 \pm 22$ nights in the hospital and $5.1 \pm 15$ nights in the other group [odds ratio (OR) 1.08; 99\% Cl: 0.92-1.28]. After two years of follow-up, more patients maintained sinus rhythm in the early rhythm control strategy group $82.1 \%$ versus $60.5 \%$ (OR 3.13; 95\% $\mathrm{Cl}: 2.55-3.84)$. No difference was observed between the groups in European Heart Rhythm Association (EHRA) class; $74.3 \%$ of patients in the early rhythm control strategy group and $72.6 \%$ in the second group were classified as EHRA class I (OR 1.14; 95\% Cl: 0.93-1.40). There was also no documented significant change in left ventricular ejection fraction values in the first and second groups (1.5 $\pm 9.8 \%$ vs. $0.8 \pm 9.8 \%$, OR 0.23; 95\% Cl: 0.46-0.91).

In conclusion, the EAST-AFNET 4 trial indicates a group of patients in whom we can think of a better preventive effect based on a rhythm control strategy using early AF ablation - it provided a better prognosis than ventricular rate control and was safe at the same time.

The first online ESC Congress will therefore be remembered not only for its pandemic-altered format but, in the authors' view, also for a large number of significant clinical trials with anticipated direct impact on management guidelines for such common conditions as heart failure, chronic coronary syndromes, and atrial fibrillation.

\section{References}

1. Imazio M, Andreis A, Brucato A, et al. Colchicine for acute and chronic coronary syndromes. Heart. 2020; 106(20): 1555-1560, doi: 10.1136/heartjnl-2020-317108, indexed in Pubmed: 32611559.

2. Nidorf SM, Eikelboom JW, Budgeon CA, et al. Low-dose colchicine for secondary prevention of cardiovascular disease. J Am Coll Cardiol. 2013; 61(4): 404-410, doi: 10.1016/j.jacc.2012.10.027, indexed in Pubmed: 23265346.

3. Nidorf SM, Fiolet ATL, Mosterd A, et al. LoDoCo2 Trial Investigators. Colchicine in patients with chronic coronary disease. N Engl J Med. 2020; 383(19): 1838-1847, doi: 10.1056/NEJMoa2021372, indexed in Pubmed: 32865380. 
4. McMurray JV, Solomon S, Inzucchi S, et al. Dapagliflozin in patients with heart failure and reduced ejection fraction. N Engl J Med. 2019; 381(21): 1995-2008, doi: 10.1056/nejmoa1911303.

5. Packer M, Butler J, Filippatos G, et al. Evaluation of the effect of sodium-glucose co-transporter 2 inhibition with empagliflozin on morbidity and mortality of patients with chronic heart failure and a reduced ejection fraction: rationale for and design of the EMPEROR-Reduced trial. Eur J Heart Fail. 2019; 21(10): 1270-1278, doi: 10.1002/ /ejhf.1536.

6. Heerspink H, Stefansson B, Chertow G, et al. Rationale and protocol of the Dapagliflozin And Prevention of Adverse outcomes in Chronic Kidney Disease (DAPA-CKD) randomized controlled trial. Nephrol Dial Transplant. 2020; 35(2): 274-282, doi: 10.1093/ndt/gfz290, indexed in Pubmed: 32030417.

7. Gheblawi M, Wang $\mathrm{K}$, Viveiros $\mathrm{A}$, et al. Angiotensin-converting enzyme 2: SARS-CoV-2 receptor and regulator of the renin-angiotensin system: celebrating the 20th anniversary of the discovery of ACE2. Circ Res. 2020; 126(10): 1456-1474, doi: 10.1161/CIRCRESAHA.120.317015, indexed in Pubmed: 32264791.

8. Position statement of the ESC Council on Hypertension on ACE-inhibitors and angiotensin receptor blockers. https://www.escardio.org/Councils/
/Council-on-Hypertension-(CHT)/News/position-statement-of-the-esccouncil-on-hypertension-on-ace-inhibitors-and-ang (October 8, 2021).

9. Lopes RD, Macedo AV, de Barros E Silva PG, et al. BRACE CORONA investigators. Continuing versus suspending angiotensin-converting enzyme inhibitors and angiotensin receptor blockers: Impact on adverse outcomes in hospitalized patients with severe acute respiratory syndrome coronavirus 2 (SARS-CoV-2) - the BRACE CORONA Trial. Am Heart J. 2020; 226: 49-59, doi: 10.1016/j.ahj.2020.05.002, indexed in Pubmed: 32502882.

10. Packer DL, Mark D, Robb R, et al. Effect of catheter ablation vs antiarrhythmic drug therapy on mortality, stroke, bleeding, and cardiac arrest among patients with atrial fibrillation. JAMA. 2019; 321(13): 12611274, doi: 10.1001/jama.2019.0693, indexed in Pubmed: 30874766.

11. Nattel S, Guasch E, Savelieva I, et al. Early management of atrial fibrillation to prevent cardiovascular complications. Eur Heart J. 2014; 35(22): 1448-1456, doi: 10.1093/eurheartj/ehu028, indexed in Pubmed: 24536084.

12. Kirchhof $P$, Camm AJ, Goette A, et al. EAST-AFNET 4 Trial Investigators. Early rhythm-control therapy in patients with atrial fibrillation. N Engl J Med. 2020; 383(14): 1305-1316, doi: 10.1056/NEJMoa2019422, indexed in Pubmed: 32865375. 